\title{
Servicio de Control Microbiológico (SCM), como Estrategia Didáctica para el laboratorio de Tecnología de Alimentos Microbiological Control Service, as Didactic Strategy for Food Technology Lab
}

\author{
Velázquez Madrazo O.C. ${ }^{1}$, E.I. Juárez Arroyo ${ }^{2}$, A. Farrés González-Saravia ${ }^{3}$, A. Cruces Martínez ${ }^{4}$, J.L. Godínez \\ Rodríguez $^{5}$, H.A. Hernández Pérez ${ }^{6}$, V. García Saturnino ${ }^{7}$. P. Severiano Pérez. ${ }^{8}$. \\ ocvm@yahoo.com, elsi.juarez.a@gmail.com, farres@unam.mx, anali94@hotmail.com,jlgzrz@hotmail.com, \\ hhernand@unam.mx, vgsline@hotmail.com,pspmex1@gmail.com
}

\author{
1. 3. 5, 7 y 8 Departamento de Alimentos y Biotecnología. $\quad \quad{ }^{2,4 y 6}$ Departamento Biología
}

Facultad de Química. Universidad Nacional Autónoma de México. Ciudad de México. México

\begin{abstract}
Resumen. Servicio de Control Microbiológico (SCM) es una Estrategia Didáctica dirigida a alumnos de Laboratorios de: Tecnología de Alimentos (LABTEC) y Microbiología de Alimentos (LMA), en la Facultad de Química (FQ) de la Universidad Nacional Autónoma de México (UNAM). Se basa en el desempeño de roles profesionales, donde alumnos de LABTEC que elaboran alimentos actúan como productores que requieren análisis microbiológicos; los alumnos de LMA actúan como proveedores del servicio de control microbiológico. Se elaboran solicitudes y reportes formales, basados en normas de referencia, para que se pueda concluir sobre la calidad higiénica del alimento. La estrategia ha permitido a los participantes, una experiencia muy cercana al ejercicio profesional, para evaluar buenas prácticas de manufactura, trabajo individual y en equipo. Todos desarrollaron habilidades de comunicación profesional, solución de problemas y conciencia sobre la importancia de la calidad higiénica de los alimentos. El control microbiológico en LABTEC se ha realizado sin recursos adicionales, pues se aplican aquellos destinados a las prácticas de LMA. Además de la experiencia de aprendizaje, se está generando mejora continua en ambas asignaturas.
\end{abstract}

Palabras clave: Competencias en Microbiología de Alimentos, Buenas Prácticas de Manufactura, Educación en Alimentos.

\begin{abstract}
Microbiological control service (SCM) as a didactic strategy is aimed to those students in laboratories of food technology (LABTEC) and food microbiology (LMA), at the Universidad Nacional Autónoma de México's School of Chemistry (FQ). It is based in professional role playing; LABTEC students elaborate the products and act as food producers in need of microbiological analysis; LMA students perform as service providers, for microbiological control. They elaborate formal requests and microbiological analysis report, based on mandatory standards, which can lead to conclusions about product's hygienic quality. The strategy has given students an experience close to professional practice, to evaluate good manufacturing practices, individual and team work. All developed professional communication skills, problems identification and proposals for solving them, as well as consciousness about the importance of hygienic quality of food. The microbiological control in LABTEC has been carried out without the need for additional resources, since those intended for LMA practices are applied to these real samples. The experience is generating continuous improvement in both courses.
\end{abstract}

Keywords: competences in food microbiology, good manufacturing practices, education in food control.

\section{INTRODUCCIÓN}

Este trabajo se llevó a cabo en la Facultad de Química de la UNAM, institución que, desde su conformación como Autónoma, así como por su tamaño y complejidad, establece: la libertad de cátedra, la promoción de interdisciplinariedad docente para la generación de nuevos conocimientos y la interacción y retroalimentación entre estudiantes y académicos, como pilares de los modelos educativos utilizados en sus diversas dependencias. Éstas abarcan desde educación media superior hasta posgrado, en gran variedad de disciplinas y especialidades científicas, tecnológicas y humanísticas, como claramente establece el Plan Educativo Nacional de la UNAM (Narro, 2012). Dicho documento plantea entre las prioridades para la Educación Superior, atender a su pertinencia y calidad.

Una de las dependencias más grandes de la UNAM es la FQ, cuya Misión es formar profesionales de excelencia con amplias capacidades en ciencia y tecnología químicas, comprometidos con aportar valor a la sociedad, en el marco del desarrollo sustentable del país.

En la carrera de Química de Alimentos, la Misión es proporcionar a la sociedad profesionales con un alto grado de competencia; mediante la formación integral de los estudiantes con personal académico de alta calidad, a través de la docencia, la investigación y la práctica profesional; contemplando tanto la adquisición de conocimientos como el desarrollo de habilidades, la capacidad para la innovación y con plena conciencia de la necesidad de actualización permanente; que coadyuven al desarrollo nacional sustentable y contribuyan a una mejor calidad de vida de la sociedad, en el área de producción de alimentos. (Facultad de Química, Plan de Desarrollo, [PDFQ], 2017).

Algunas de las metas del PDFQ (FQ, 2017) en esta licenciatura son: Propiciar la mejora continua del plan de estudios de la carrera aprovechando su orientación a la ciencia aplicada a la tecnología y a la investigación; proporcionar a los estudiantes del programa una formación integral de alta calidad basada en las ciencias, dirigida claramente a la solución de los problemas nacionales y fomentar la participación colegiada de todos los departamentos 
académicos de la Facultad de Química en los procesos de mejora continua del programa.

\section{A. Modelos educativos}

\section{La Estrategia SCM se basa en dos importantes modelos:}

- la teoría de desarrollo cognitivo de Vigotsky, que da especial valor al entorno cultural (y profesional, como parte de la cultura vigente, aprendida por los futuros profesionales) y a la interacción social (Acosta, 2006). En este caso la interacción social abarca, además de los profesores, a los compañeros de la otra asignatura y a los enlaces de Servicio Social;

- el desarrollo de competencias profesionales, consideradas como la capacidad de poner en práctica un conjunto de comportamientos que dan lugar a la realización de una actividad o tarea con un mínimo nivel de calidad y eficacia. En el ejercicio de la competencia, se requiere la presencia de otros elementos: el saber y el saber hacer (conocimientos y habilidades), el saber estar (actitud acorde con la acción), el querer hacer (motivación) y el poder hacer (características de personalidad e inteligencia compatibles), así como condiciones contextuales favorecedoras de la acción (Pérez, 2006).

\section{B. La ZDP de Vigotsky}

La teoría de Vigotsky, establece que el aprendizaje se da siempre en medio de una situación social y está fuertemente determinado por ésta; dicha situación social se ve favorecida en lo que Vigotsky llama la zona de desarrollo proximal (ZDP). Ésta se define como la oportunidad de aprendizaje que se da en la interacción entre un aprendiz con cierto bagaje, pero no-competente en el contenido por abordar, y una persona competente en la tarea, dispuesta a guiar dicho aprendizaje; y considera que en esta situación, el aprendiz se vuelve competente e independiente respecto a la tarea, gracias a la interacción. El concepto es bastante complejo pero implica, además del aprendizaje asistido, la posibilidad de que éste sea logrado de manera independiente por el aprendiz, aunque con mayor dificultad y/o menor eficiencia. (Chaiklin, 2003).

En el mismo documento, Chaiklin (2003) hace diversas precisiones sobre la ZDP:

a. En la ZDP la enseñanza-aprendizaje debe enfocarse en procesos de maduración psicológica, no en la práctica repetitiva de habilidades existentes.

b. el contenido y significancia de la ZDP cambia conforme va cambiando el desarrollo del estudiante y conforme se aproxima a otras etapas de madurez; para fines de este trabajo, la transición al campo profesional.

c. la función teórica de la ZDP de Vigotsky se refiere a la maduración y desarrollo integral del aprendiz, no al desempeño en una sola tarea. Por eso el apoyo del maestro (o individuo más competente) es útil para la maduración y transición a etapas diferentes.

El desarrollo de la estrategia SCM favorece la maduración psicológica, más allá de la práctica no repetitiva, por la interacción entre los alumnos de $6^{\circ}$ semestre (LMA, proveedores de servicios analíticos) y los de $8^{\circ}$ (LABTEC, productores de alimentos que requieren los análisis), por la correlación de áreas de ejercicio y por la semejanza de la situación de aprendizaje, con la práctica profesional.

Finalmente, para favorecer la maduración y el desarrollo integral, se cuenta con profesores y enlaces de Servicio Social, como individuos competentes para guiar al alumno a través de la ZDP. Como los alumnos tendrán esta experiencia en las dos asignaturas, con un año de diferencia y ejerciendo diferentes papeles, la oportunidad de maduración de los estudiantes, se incrementa (García Retana, 2011). Al manejar una situación muy parecida a la de la realidad laboral, se generan oportunidades y herramientas que permiten a los estudiantes, resolver problemas, evaluar el trabajo individual y en equipo, superar debilidades y optimizar fortalezas (Curwin, 2014).

\section{El desarrollo de Competencias Profesionales}

El aprendizaje de competencias se basa en eventos cognitivos y no cognitivos y requiere de varios contextos en los que se combinen situaciones formales e informales de aprendizaje (Barth y Godemann, 2007), algunas de la cuales deben ser muy semejantes a aquellas en las que se ejerce la competencia profesional buscada, lo cual se ha complicado en los planes de estudios que fragmentan el conocimiento en asignaturas poco conectadas (Ruiz y Pachano, 2005).

La participación comprometida de los estudiantes en experiencias educativas integrales que les permitan transformar su propio aprendizaje y adquirir las competencias profesionales ha resultado muy interesante en varios intentos de mejorar la calidad de la educación superior. Entre estas competencias profesionales, está la capacidad de crítica dentro del compromiso con la sociedad, a través de valores como la solidaridad humana y el respeto; este aprendizaje no se transmite de manera tradicional, sino se desarrolla mediante el trabajo innovador en actividades de investigación, extensión y de debate académico (Ruiz y Pachano, 2005). Por ello, una de las líneas de trabajo dentro del esfuerzo mejora continua de la licenciatura en Química de Alimentos, es el desarrollo de competencias profesionales que, por ser habilidades holísticas, integrales e integradoras, requieren para lograrse, de actividades de aprendizaje con estas características.

En este contexto, la producción de alimentos procesados (como sucede en LABTEC) y la generación de un dictamen sobre la calidad de un producto elaborado por terceros (como sucede en LMA), resultan complementarias y muy útiles. Mediante el trabajo de laboratorio con metas específicas y realistas en ambas asignaturas, como plantea el SCM, la relación profesor-alumno y las relaciones alumno-alumno, son más personales y prevalece la actividad del alumno sobre la del profesor; en el laboratorio se desarrollan diversas habilidades, se adquieren conocimientos y aptitudes generando competencias cognoscitivas, psicomotrices y afectivas, así como interés y satisfacción personal; destacan las competencias para integrar datos de diversas fuentes, como origen, proceso, parámetros fisicoquímicos, resultados microbiológicos y otros, para detectar oportunamente problemas y oportunidades (Díaz y cols, 2015).

Varios autores incluyen las competencias del trabajo en equipo, o colaborativo como algunas de las competencias profesionales más importantes en estos tiempos. En educación, 
el trabajo colaborativo ofrece ventajas para el logro de competencias profesionales, por ejemplo por la rotación de funciones en el equipo de trabajo, ya que favorece la comprensión de los diferentes aspectos de la actividad profesional y de su integración. Otras ventajas son la práctica comunicativa y la retroalimentación informal pero asertiva. (González y Díaz Matajira, 2005). El aprendizaje colaborativo ayuda al estudiante a enfrentar su autonomía y el conocimiento no fundacional al que será expuesto; implica que se elabora un constructo social para resolver el problema o para interpretar la realidad, entre pares.

La adquisición de competencias profesionales se considera importante porque éstas permiten hacer frente a contextos laborales cambiantes en los que la polivalencia y la flexibilidad son necesarios, y porque consideran dos referentes básicos: el social y el individual (Navío, 2005), lo que coincide con una de las metas específicas del Plan de Educación vigente en la UNAM (Narro y cols, 2012).

\section{Juego de roles}

Finalmente, el juego de roles o el desempeño de papeles específicos, es una técnica ampliamente utilizada en ciencias sociales, para comprender contenidos teóricos; pero también se ha aplicado en Ciencias naturales y exactas, con muy buenos resultados (Howes y Cruz, 2009). En este trabajo se usan los roles profesionales, para dar a la estrategia SCM, mayor semejanza con la realidad y para motivar a los estudiantes.

\section{E. Evaluación}

En el modelo de Vigotsky, sobre todo cuando se trabaja en la ZDP, siempre deben considerarse los logros del estudiante respecto al modelo o a los logros teóricos para la actividad o tema, lo que implica un énfasis en la coevaluación profesoralumno y en la retroalimentación constructiva para que el estudiante logre objetivos de aprendizaje y el docente comprenda y mejore su propio ejercicio profesional (Chaiklin, 2003).

Tobón (2013) reitera que la evaluación tiene un papel muy importante en el proceso y que debe abordarse mediante un esquema dinámico e integral, que permita clarificar el proceso. Barth \& Godemann (2007), al hablar de competencias en la Educación Superior, establecen que el desarrollo de las mismas tiene que entenderse también como aprendizaje de valores, lo que implica proceso de interiorización, recepción, reproducción y comunicación de valores, como puntos centrales del proceso. El componente afectivo debe estar presente en las competencias para permitir un adecuado desempeño y, sobre todo, la actitud crítica para detectar posibles acciones que sin duda va a requerir el estudiante cuando ejerza en la vida profesional.

Así pues, para fines de este trabajo, la evaluación es una herramienta que busca la mejora continua del estudiante y del proceso; no es un mecanismo de medición. Siguiendo las consideraciones de García Retana (2011), el docente, lejos de acreditar o suspender, ayuda al estudiante a lograr el desempeño esperado, a superar obstáculos, mientras aprende de su ejercicio, de sus recursos, de sus alumnos y su ambiente, para mejorar continuamente su desempeño y su entorno. La evaluación es continua, integral, aplicada a la retroalimentación y al mejoramiento, en todo momento.
Tanto el Plan de diez años para desarrollar el Sistema Educativo Nacional (Narro y cols, 2012) como el Plan de desarrollo Institucional 2015-2019, del actual Rector de la UNAM, E. Graue (2017) dan especial importancia a la evaluación continua e integral y a la retroalimentación como fundamento de la mejora continua de las instituciones, lo que hace de este trabajo un instrumento para lograrlo, en nuestro ámbito.

\section{CONTEXTO}

Entre los principales objetivos del Plan de Desarrollo de la Coordinación de Carrera de Química de Alimentos en la FQ de la UNAM están:

- Proporcionar a los estudiantes una formación integral de alta calidad basada en las ciencias, dirigida claramente a la solución de los problemas nacionales desde el origen de la cadena de abasto de los alimentos hasta sus efectos a corto y largo plazo sobre la sociedad y

- Propiciar la formación integral de los estudiantes de Química de Alimentos al promover el contacto del estudiante con instituciones académicas, institutos nacionales y sector productivo, con el fin de ofrecer la participación en proyectos que podrían culminar en el desarrollo de su tesis profesional.

Este último objetivo, en particular aplica a los alumnos de LABTEC, que pueden empezar proyectos de tesis experimentales en el siguiente semestre, motivados por los resultados de esta estrategia; desde luego, también aplica a los de LMA, aunque a mayor plazo.

\section{A. LMA}

Laboratorio de Microbiología de Alimentos (LMA) es una materia que se imparte en el sexto semestre de la carrera de Química de Alimentos. En este curso, los alumnos aprenden los fundamentos teóricos y las técnicas básicas para determinar la presencia de microorganismos en alimentos, específicamente los microorganismos indicadores y los patógenos considerados en las especificaciones de las Normas Oficiales Mexicanas (NOM), así como determinaciones importantes para establecer la calidad del alimento, en general. También hacen estudios sobre higiene de superficies, vivas e inertes y sobre el ambiente.

Los resultados obtenidos se utilizan para establecer calidad higiénica, comenzando por señalar la conformidad o la noconformidad del producto, con especificaciones de las NOM.

\section{B. LABTEC}

Laboratorio de Tecnología de Alimentos (LABTEC) es una materia que se imparte a los alumnos que cursan octavo semestre de la carrera de Química de Alimentos, en la FQ de la UNAM. Esta materia consta de cinco módulos en dónde se integran los conocimientos adquiridos en semestres anteriores. Los módulos son: Cárnicos, Lácteos, Cereales, Vegetales y Diseño de Nuevos Productos. En cada uno de los módulos se aplican diferentes tecnologías para la elaboración de productos alimenticios, de acuerdo con los objetivos del programa. En todos los módulos, se realizan pruebas de calidad para caracterizar materia prima y producto terminado. Se aplican 
las tecnologías, y los productos obtenidos se someten a evaluación sensorial y físico-química.

Los resultados se integran en un reporte para concluir, entre otras cosas, si se cumplen o no, las especificaciones de las NOM en materia de alimentos (Curwin, 2014). Aunque hay limitaciones en el equipo y espacio, también hay ventajas como el manejo de cantidades pequeñas y el nivel educativo de los estudiantes, por lo que se espera que obtengan productos conformes con las NOM y de calidad.

\section{DESCRIPCIÓN}

Este es un estudio exploratorio y descriptivo, sobre la aplicación de los modelos mencionados para lograr el desarrollo de competencias profesionales a través de una experiencia que integra a alumnos de dos asignaturas y semestres diferentes.

Se ha llevado a cabo a lo largo de tres semestres lectivos (más uno de preparación, dedicado a generar el proyecto y capacitar a los docentes y enlaces), en cada uno de los cuales han participado tres grupos de LABTEC y 5 de LMA, así como casi 20 profesores involucrados en los mismos y 15 enlaces de Servicio Social. Los estudiantes que han participado son, en promedio 90 de LABTEC y 120 de LMA, cada semestre.

\section{Los objetivos del proyecto han sido:}

- Retroalimentar a los alumnos de LABTEC con parámetros microbiológicos que les permitan concluir sobre inocuidad, calidad y estabilidad de sus productos, y sobre su desempeño en la producción.

- Proveer a los alumnos de LMA con unidades de prueba directas de planta (LABTEC), para realizar análisis microbiológico en materia prima y producto terminado, incluyendo seguimiento.

- Desarrollar en alumnos de ambas asignaturas, competencias de comunicación profesional, de interpretación de informes y resultados analíticos, y de toma de decisiones basadas en resultados analíticos.

- Impulsar aprendizaje integral, concientización oportuna en LABTEC y concientización previa en LMA, sobre el papel que tiene el desempeño personal de cada uno, en el resultado final de la producción de alimentos.

La figura 1, en la siguiente columna, muestra en un diagrama las etapas que involucra la estrategia SCM señalando ambos laboratorios (LABTEC y LMA), así como los enlaces de Servicio Social que facilitan la aplicación mediante intercambio de información, muestras y formatos. Los enlaces de Servicio Social son alumnos más avanzados o al menos, en $8^{\circ}$ semestre, que han estado trabajando en microbiología de alimentos al menos durante un semestre, por lo que constituyen un buen apoyo para sus compañeros, específicamente para ayudarlos a aprovechar la experiencia en la ZDP, de acuerdo con el modelo de Vigotsky. Esta colaboración aunada a la participación de los docentes y a los aprendizajes teóricos previos, ayuda a asegurar un buen trabajo en LMA, así como una buena interpretación, identificación de problemas y, sobre todo, generación de buenas soluciones, en ambas asignaturas.
Finalmente, como muestra el esquema, la estrategia completa incluye el análisis de la información registrada: muestras, procesos, equipo utilizado, alumnos productores, materias primas, resultados microbiológicos, frecuencias de los hallazgos, etc. Y lo importante es que, a través de este análisis posterior de datos, se han identificado importantes áreas de oportunidad, por ejemplo en la sanitización del equipo de producción, y se ha generado material didáctico, específicamente varios “casos de estudio” que se pueden usar en estas dos asignaturas, así como en Procesos de Alimentos, Calidad y otras asignaturas.

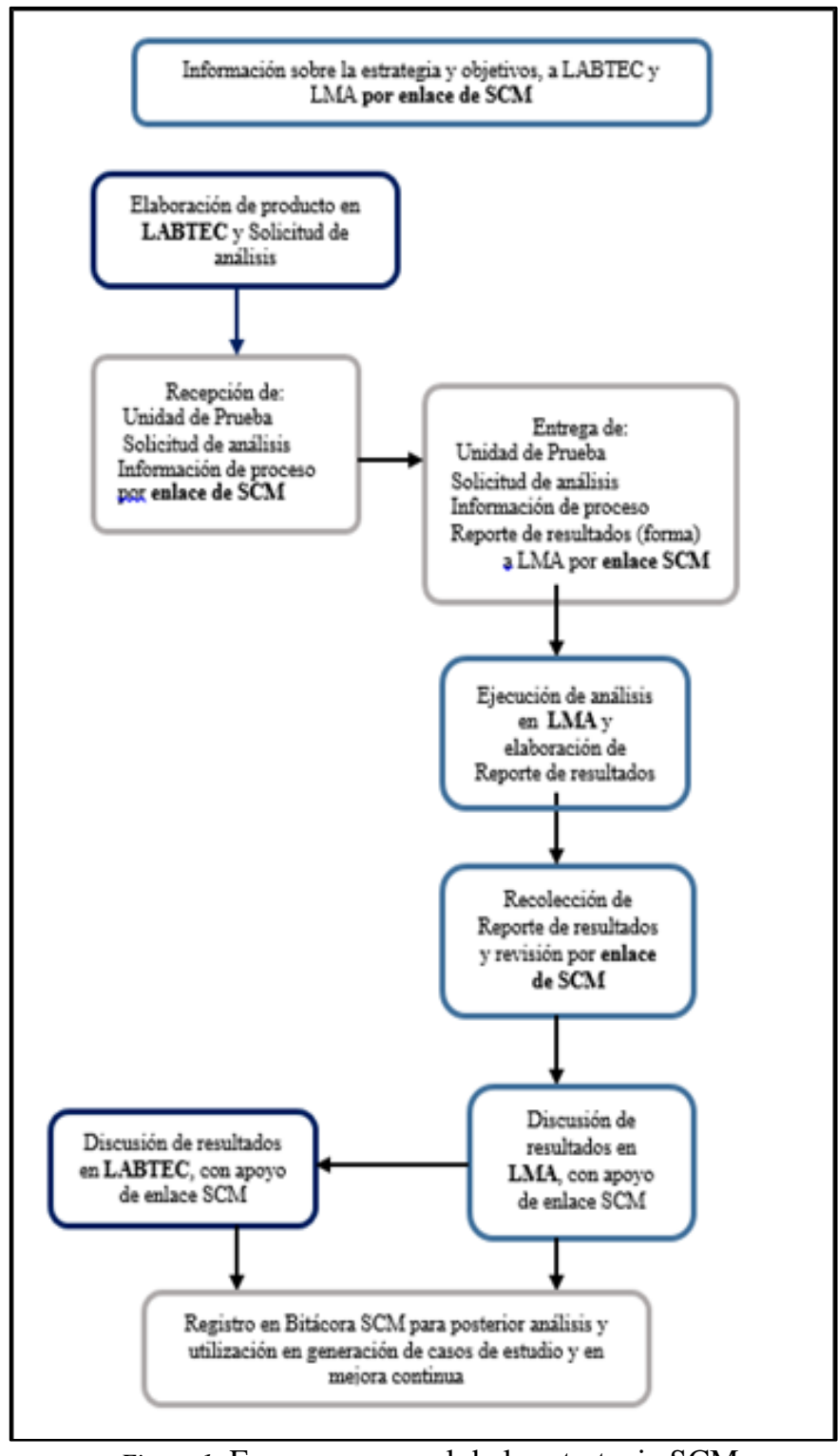

Figura 1. Esquema general de la estrategia SCM

El mecanismo de esta estrategia consiste en proporcionar a los estudiantes una experiencia similar a lo que sucede en una planta de producción de alimentos (OECD, 2015). Así, los alumnos de LABTEC, como productores de alimentos procesados, solicitan formalmente a los alumnos del LMA, que como analistas del Laboratorio de Servicio de Microbiología, lleven a cabo los análisis microbiológicos de materias primas y de producto terminado. Todas las muestras son codificadas y manejadas de manera formal. Desde luego, 
se diseñaron formatos de solicitud de análisis y de reporte de resultados y se han modificado conforme a la experiencia al aplicar el programa.

Para tener réplicas de los análisis, los maestros distribuyen las muestras a dos o tres equipos en cada caso. Un coordinador de analistas, que es un estudiante cubriendo Servicio Social, procesa los reportes de cada equipo del LMA para integrar los resultados de los duplicados o triplicados y los organiza en un reporte formal de resultados, que es revisado y aprobado por los profesores de LMA y entregado a los profesores y alumnos de LABTEC. En la sesión de cierre de cada módulo de LABTEC (Cárnicos, Lácteos, Cereales, Vegetales y Diseño de Nuevos Productos) se discuten estos resultados, con énfasis en las competencias de interpretación, detección de problemas y propuestas de solución. En LMA se discuten los resultados y problemas de la determinación, así como las sugerencias adicionales enviadas a LABTEC.

Las unidades de prueba correspondientes al módulo Cárnicos LABTEC fueron: carne cruda, condimentos y aditivos para elaborar; chorizo curado, salchicha cocida y chuleta ahumada. En el módulo de Lácteos se analizaron: leche bronca, leche pasteurizada, crema, queso fresco y queso madurado. Las unidades de prueba del módulo de Cereales fueron: semolina, harina de trigo, masa de nixtamal y pastas. En el módulo Vegetales se analizaron: jugos pasteurizados, verduras en salmuera, y frutas en almíbar (mango y durazno). Las tecnologías aplicadas a estos productos fueron: curado y ahumado de carnes, pasteurización continua y por lotes, extrusión, envasado en caliente, enlatado y esterilización comercial. Todas son metodologías tradicionales descritas en el manual de prácticas de LABTEC, editado por el Departamento de Alimentos y Tecnología de la Facultad de Química, UNAM. El análisis de superficies incluyó: picadora de carne, molino de carne, extrusor de pastas, pasteurizador, refrigeradores, estufas, ollas, ambiente (aire y agua), manos de alumnos LABTEC.

Los grupos de LMA realizaron las determinaciones de: microorganismos indicadores (Mesófilos aerobios, Mohos y Levaduras, Coliformes totales, Escherichia coli), y patógenos (Salmonella spp., Vibrio cholerae, Staphylococcus aureus enterotoxigénico), utilizando metodologías tradicionales (descritas en las NOM) y métodos rápidos autorizados (Placas Petrifilm ${ }^{\mathrm{TM}}$, Placas CompactDry® y Bioluminiscencia).

$\mathrm{Al}$ integrar el reporte se enfrenta la dificultad de resultados diferentes entre los analistas de LMA, que se resuelve a partir del estatus de la muestra (conforme o no-conforme), más que a partir de los números; este enfoque funciona bastante bien para integrar los reportes, aunque ocasionalmente es imposible concluir sobre alguna muestra (menos de 2\%) (Curwin, 2014).

\section{Resultados}

Se consideran no-conformes los productos o materias primas que incumplen una o más especificaciones microbiológicas de la NOM respectiva, independientemente del nivel. Las no-conformidades se han reducido a menos de la mitad, en cada semestre en que se ha implementado una medida de mejora: ejercicio inicial de sensibilización en uno, asesoría para muestreo en otro y procesos operativos estandarizados de sanitización (POES) en el último.
La calidad de los reportes de LABTEC ha mejorado, llevando las calificaciones promedio de 7.9 a 9.0, calificando con los mismos indicadores; el promedio de calificación en las actividades de solicitud de análisis y reporte de resultados ha subido de 6.3 a 8.4 en promedio.

Se han generado dos ejercicios adicionales para sensibilización y control de procesos en LABTEC: 6 casos de estudio para utilizar en éstas y otras asignaturas y 5 POES para equipo y material de limpieza. Al ser ésta una estrategia didáctica, el énfasis en el análisis de resultados en ambas asignaturas se dirige a identificar causas de no-conformidad y especialmente, medidas para resolver los problemas. Al comparar los reportes de semestres anteriores a la implementación de la estrategia, con algunos de los más recientes, se aprecia una diferencia en el análisis de resultados, que es más completo y fundamentado a partir de la implementación de la estrategia, y en las conclusiones, que son más claras y acertadas, incluyendo la crítica sobre su propio desempeño, lo que contribuye a la metacognición (Maats \& O’Brien, 2015).

\section{CONCLUSIONES}

La estrategia didáctica SCM ha funcionado para generar una experiencia práctica muy similar al ejercicio profesional, para los alumnos de LABTEC y de LMA. La retroalimentación para ambos ha sido útil más allá de los objetivos de aprendizaje, pues ha generado competencias para el trabajo y conciencia sobre la importancia de su propio trabajo (Maats \&O’Brien, 2015; OECD, 2015). Además se está logrando controlar la efectividad de las tecnologías aplicadas en LABTEC y la reproducibilidad de los análisis realizados en LMA.

Se empieza a percibir, aunque hará falta estudiar el tema por más tiempo, que los alumnos participantes de LMA ponen más atención a las medidas preventivas y al control de procesos, cuando cursan LABTEC, en comparación con los estudiantes que cursaron LMA sin participar en la estrategia SCM. Sin duda, el seguimiento de resultados permitirá encontrar otras oportunidades para la mejora continua, en ambos laboratorios.

\section{AgRADECIMIENTOS}

Este proyecto se llevó a cabo con apoyo de la Dirección General de Personal Académico (DGPA) de la UNAM mediante el PAPIME 204516 aprobado para 2016 y 2017. Los autores agradecemos este apoyo.

También agradecemos la participación de los colegas profesores del LMA, de LABTEC, el apoyo de los jefes de los Departamentos de Biología (Dr. R. Pastelín Palacios) y de Alimentos y Biotecnología (Dr. M. Gimeno Seco), así como de todos los estudiantes de Servicio Social que han apoyado el trabajo, especialmente Concepción Sánchez Paredes y Paola Lozano Rodríguez.

\section{REFERENCIAS}

Acosta Rodríguez, M. (2006). El aprendizaje visto como un proceso de interacción social. La perspectiva Vigotskianavista desde la complejidad. Rev. Ciencias de la 
Educación. Año 6, 1(27 ene-jun), 123-134. Disponible en: http://servicio.bc.uc.edu.ve/educacion/revista/volIn27/278.pdf

Barth, M., J. Godemann, M. Rieckmann and U. Stoltenberg, (2007),"Developing key competencies for sustainable development in higher education", International Journal of Sustainability in Higher Education, 8(4), 416 - 430. http://dx.doi.org/10.1108/14676370710823582

Chaiklin, S. (2003). The zone of proximal development in Vygotsky's analysis of learning and instruction. In Kozulin, Gindis, Ageyev, and Miller. (Ed.). Vygotsky's educational theory and practice in cultural context. (pp. 39-64). Cambridge, U.K.: Cambridge University Press.

Curwin, R. 2014 (Oct 28 ${ }^{\text {th }}$ ). It's a mistake not to use mistakes as part of the learning process. George Lucas Educational Foundation. Disponible a través de Edutopia en: https://www.edutopia.org/blog/use-mistakes-in-learningprocess-richard-curwin

Díaz, J.P., A. R. Bar y M.C. Ortiz. 2015 (oct-dic). La lectura crítica y su relación con la formación disciplinar de estudiantes universitarios. Revista de la Educación Superior. 44(oct-dic), 139-158. Disponible a través de Science Direct en: http://www.sciencedirect.com/science/article/pii/S0185276 015001223

Facultad de Química. (2017). Plan de Desarrollo. Licenciatura en Química de Alimentos. Enseñanza. Disponible a través de:_https:/quimica.unam.mx/ensenanza/licenciaturas-dela-facultad-de-quimica/quimica-de-alimentos/

García Retana, J.A. (2011). Modelo educativo basado en competencias: Importancia y necesidad. Actualidades Investigativas en Educación. 11(3),1-24. Disponible en: http://www.redalyc.org/articulo.oa?id=44722178014

González, G. y L. Díaz Matajira. (2005). Aprendizaje colaborativo: una experiencia desde las aulas universitarias. Educación y Educadores (Colombia). 8:2144.

Graue W, E. (2017). Plan de Desarrollo Institucional 20152017. Universidad Nacional Autónoma de México. México.
Howes, E.V. and B.C. Cruz (2009), Role-Playing in Science Education: An Effective Strategy for Developing Multiple Perspectives. Journal of Elementary Science Education. 21(3): 33-46. http://www.jstor.org/stable/43156175

Maats, H. \& K. O’Brien. 2015 (Apr 21 ${ }^{\text {st }}$ ). Hands-Off teaching Cultivates Metacognition. Teaching Strategies, George Lucas Educational Foundation. Disponible a través de Edutopia en: https://www.edutopia.org/blog/hands-offteaching-cultivates-metacognition-hunter-maats-katieobrien

Narro Robles, J.; J. Martuscelli Quintana y E. Bárzana García (Coord.). (2012) Plan de diez años para desarrollar el Sistema Educativo Nacional. [En línea]. México: Dirección General de Publicaciones y Fomento Editorial, UNAM._http://www.planeducativonacional.unam.mx

Navío Gámez, A. (2005). Propuestas conceptuales en torno a la competencia profesional. Revista de Educación. (España), (337), 213-234.

OECD - Gobierno de España. 2015. Informe de diagnóstico de la Estrategia de Competencias de la OCDE: España. Gabinete de la Secretaría de Estado de Educación, Formación Profesional y Universidades, Ministerio de Educación, Cultura y Deporte. Disponible a través de Internet en: http://www.oecd.org/skills/nationalskillsstrategies/Spain_ Diagnostic_Report_Espagnol.pdf

Pérez, J. C. (2006). La orientación profesional y la evaluación desde el enfoque de competencias. Revista Mexicana de Orientación Educativa, 2a ${ }^{a}$ Época. 4 (8), 2-13

Ruiz, L.F., Pachano, L. (may-ago 2005). Modelo teórico de evaluación constructivista orientado hacia el desarrollo de competencias en el estudiante universitario. Encuentro Educacional. (Venezuela). 12 (2), 230-242.

Tobón, S. (208)._L La Formación basada en competencias en la Educación Superior: El enfoque complejo. Curso Iglu. Universidad Autónoma de Guadalajara. México. 